APL in press

\title{
Coherent Exciton Lasing in ZnSe/ZnCdSe Quantum Wells?
}

\author{
M.E. Flatté, E. Runge* and H. Ehrenreich \\ Division of Applied Sciences, Harvard University, Cambridge, Massachusetts 02138
}

(July 20, 1994)

\begin{abstract}
A new mechanism for exciton lasing in $\mathrm{ZnSe} / \mathrm{ZnCdSe}$ quantum wells is proposed. Lasing, occurring below the lowest exciton line, may be associated with a BCS-like condensed (coherent) exciton state. This state is most stable at low temperatures for densities in the transition region separating the exciton Bose gas and the coherent exciton state. Calculations show the gain region to lie below the exciton line and to be separated from the absorption regime by a transparency region of width, for example, about $80 \mathrm{meV}$ for a $90 \AA \mathrm{ZnSe} / \mathrm{Zn}_{.75} \mathrm{Cd}_{.25}$ Se quantum well. Experimental observation of the transparency region using differential spectroscopy would confirm this picture. PACS: 78.66.Hf, 71.35.+z, 78.45.+h
\end{abstract}


Time-resolved pump-probe measurements of absorption in $\mathrm{ZnSe} / \mathrm{ZnCdSe}$ quantum wells indicate that lasing in these systems may originate from exciton states [1] 3]. Excitons in this system are unusually robust; the binding energy (40 meV in a $90 \AA$ quantum well) exceeds both $k_{B} T$ at room-temperature and the LO-phonon energy (31 meV). This situation sharply contrasts that in GaAs, where the quantum-well-enhanced binding energy can be $10 \mathrm{meV}$, and the LO-phonon energy is $36 \mathrm{meV}$. Carriers in the ZnSe/ZnCdSe systems relax quickly to quasi-equilibrium distributions due to LO-phonon coupling.

Photon emission in this system exhibits two unusual characteristics: emission occurs at energies below the exciton line and at densities for which the excitons can no longer be regarded as independent. As a criterion for the importance of many-body exciton interactions, the characteristic exciton density $n_{o}$ is most simply defined as that for which the binding energy of an effective exciton in a Bose gas vanishes. In the presence of an increasing density of other excitons, the exciton wave functions begins to overlap. At low temperatures this overlap marks the transition to the coherent state described below, while at higher temperatures it marks the transition to an electron-hole plasma. Our value for $n_{o}$ agrees roughly with that expected on the basis of a Mott criterion. Both values are an order of magnitude smaller than a criterion used by Nurmikko [1-3] based on densities when isolated excitons, characterized by density-independent Bohr orbits, first overlap. This letter will show how an excitonic picture survives, with modifications, at such high densities and how emission occurs below the exciton line.

The mechanism suggested by Nurmikko et al. in this seminal work [1] requires an inhomogeneous exciton linewidth due to imperfections which substantially exceeds the homogeneous linewidth. The exciton states in their model are non-overlapping and have a density below the electron-hole plasma transition. The occupied states lie at the bottom of the inhomogeneous exciton band. As a result lasing involves excitons lying below the center of the exciton line.

This mechanism could not be operative in the absence of crystal imperfections. We propose here a new mechanism for lasing in the ZnSe/ZnCdSe quantum wells which does 
not rely on disorder. It is based on an unusual state of the interacting electron-hole system which involves a condensation of electron-hole pairs [4], similar to the BCS condensation of electron-electron pairs in a superconductor. More precisely, at very low, moderate, and higher densities it corresponds respectively to independent excitons, a Bose condensate and the BCS state respectively. We shall refer to the last of these as the "coherent exciton" state. The smooth transition from the bosonic to the BCS state occurs near the characteristic density $n_{o} \approx 4 \times 10^{11} \mathrm{~cm}^{-2}$ in a $90 \AA \mathrm{ZnSe} / \mathrm{Zn}_{.75} \mathrm{Cd}_{.25}$ Se quantum well.

A heuristic sketch of this state is shown in Fig. 1 for the characteristic density $n_{o}$. Figure 1a shows the non-interacting electron-hole plasma at $0 \mathrm{~K} ; \mu_{e}$ and $\mu_{h}$ are the electron and hole quasi-Fermi levels respectively. This non-equilibrium state is induced by a forward bias across the active region. The Hamiltonian of this system is

$$
H=\sum_{\mathbf{k}} \epsilon_{c}(k) c_{c, \mathbf{k}}^{\dagger} c_{c, \mathbf{k}}+\sum_{\mathbf{k}} \epsilon_{v}(k) c_{v, \mathbf{k}}^{\dagger} c_{v, \mathbf{k}}
$$

where $c_{c, \mathbf{k}}^{\dagger}\left(c_{v,-\mathbf{k}}\right)$ creates a conduction electron (valence hole) with momentum $\mathbf{k}$. Spin indices and sums are implied. Here

$$
\epsilon_{c}(k)=\frac{k^{2}}{2 m_{e}}+E_{G}-\mu_{e}, \quad \epsilon_{v}(k)=-\frac{k^{2}}{2 m_{h}}+\mu_{h}, \quad \mu_{e}+\mu_{h}=E_{G}
$$

and $E_{G}, m_{e}$ and $m_{h}$ are the band gap and effective masses respectively. In the presence of the electron-hole interaction,

$$
H_{e-h}=-\sum_{\mathbf{q}, \mathbf{k}, \mathbf{k}^{\prime}} V(q) c_{c, \mathbf{k}+\mathbf{q}}^{\dagger} c_{v \mathbf{k}^{\prime}}^{\dagger} c_{v, \mathbf{k}^{\prime}+\mathbf{q}} c_{c, \mathbf{k}}
$$

a quasi-particle gap forms at the conduction-electron and valence-hole Fermi surfaces (shown in Fig. 1b). Here $V(q)$ is the attractive effective electron-hole Coulomb interaction modified by screening due to the exciton polarizability. For our calculations we use a stateindependent $V(q)$ whose magnitude and functional form is determined by the exciton model specified below.

The assumption of singlet pairing between electrons and holes having momentum $\mathbf{k}$ and -k respectively leads to a gap equation [4]: 


$$
2 \Delta(k)=\sum_{q} V(q) \frac{2 \Delta(k+q)}{E_{e h}(k+q)}
$$

Here

$$
E_{e h}(k)=\sqrt{\left(\epsilon_{c}(k)-\epsilon_{v}(k)\right)^{2}+4|\Delta(k)|^{2}}
$$

and $\Delta(k)$ is analogous to the gap parameter in the BCS theory. The number of excitons in a quantum well having unit volume is

$$
n=\sum_{k}\left(\frac{1}{2}-\frac{\left(\epsilon_{c}(k)-\epsilon_{v}(k)\right)}{2 E_{e h}(k)}\right) \quad .
$$

The pairing assumption used here is analogous to the pairing assumption in superconductivity involving spin up electrons of momentum $\mathbf{k}$ and spin down electrons of momentum -k. The gaps in the valence and conduction band labelled $E_{e h} / 2$ in Fig. $1 \mathrm{~b}$ each correspond to half the energy $E_{e h}$ required to break up the exciton, one half being assigned to the electron $e$ and hole $h$ respectively. [5]

The sketch in Fig. 1b satisfies the condition $\mu_{e h}=E_{G}$ defining the characteristic density $n_{o}$. The valence-conduction band gap is seen to be smaller than the corresponding quantity $E_{G}$ of Fig. 1a because of band gap renormalization effects associated with the electron-hole Coulomb interaction. (The corresponding electron-electron and hole-hole interactions neglected here would further increase this gap shrinkage). The gain and absorption regions are illustrated by the arrows marked "g" and "a" respectively. The gaps $E_{\text {eh }}$ produce a transparency regime, illustrated by the arrow "tr", separating the two regions. (The transparency regime width $2 E_{e h}$, whose magnitude is of the order of the exciton binding energy, should be experimentally observable if this model is applicable to the present situation.) In addition, the enlarged density of states near the gap edge enhances the gain near the transparency edge. These features are qualitatively consistent with the observed lasing spectrum in the $\mathrm{ZnSe} / \mathrm{ZnCdSe}$ quantum wells at energies well below the center of the exciton line [1 3 .

Equation (4) has been solved for $\Delta(k)$ for $30 \AA$ and $90 \AA$ quantum wells as well as the idealized two-dimensional case. The results are shown in Fig. 2. Since the heavy-hole, lighthole band splitting is substantial in these systems (79 meV in the $90 \AA$ case), the independent 
sub-band model and the rod model, used by Young et. al. [7] are valid. These approximations have been used in quantitatively accurate calculations of exciton binding energies and optical absorption coefficients in III-V [8] and II-VI [7] superlattices and quantum wells. In the rod model, the interaction potential is approximated by considering the electron and hole to be rods having length equal to the well width. Screening effects of $V(q)$ due to the presence of other excitons are found to be small within this approximation, particularly for the $90 \AA$ quantum well, and will be neglected.

The fundamental absorption coefficient in the coherent state is given by [6]

$$
\begin{gathered}
\alpha(\omega)=\frac{2 \pi^{2} e^{2} \hbar}{n m c} \frac{2}{m \hbar \omega} \sum_{\mathbf{k}}\left|\mathbf{e} \cdot \mathbf{p}_{c, v}(\mathbf{k})\right|^{2}\left[-\left(\frac{1}{2}-\frac{\left(\epsilon_{c}(k)-\epsilon_{v}(k)\right)}{2 E_{e h}(k)}\right)^{2} \delta\left(\omega-\mu_{e h}+E_{e h}(k)\right)\right. \\
\left.+\left(\frac{1}{2}+\frac{\left(\epsilon_{c}(k)-\epsilon_{v}(k)\right)}{2 E_{e h}(k)}\right)^{2} \delta\left(\omega-\mu_{e h}-E_{e h}(k)\right)\right] .
\end{gathered}
$$

Here $n$ is the index of refraction, $\mathbf{e}$ is the photon's polarization, and the momentum matrix element $\mathbf{p}_{c, v}(\mathbf{k})$ is obtained from band-structure calculations using a $\mathbf{k} \cdot \mathbf{p}$ method [7,8].

Figure 3 shows the gain spectrum for a $90 \AA$ ZnCdSe quantum well laser for three carrier densities as a function of the photon energy $h \omega$ relative to $\mu_{e h}$. The characteristic density is $n_{o}=4 \times 10^{11} \mathrm{~cm}^{-2}$. The curves have been assigned a linewidth of $10 \mathrm{meV}$,

corresponding to that measured at low temperatures [5], which is presumably associated with inhomogeneous broadening. Each curve shows a sharply peaked gain region and a corresponding absorption region below and above $\mu_{e h}$ respectively. In a collisionless ideal quantum well these regions would be separated by a transparency region of width $2 E_{e h}$ around $\mu_{e h}$. This feature is somewhat blurred by the phenomenological linewidth. By contrasting the energy gain at $3.7 \times 10^{11} \mathrm{~cm}^{-2}$ with that at $4.1 \times 10^{11} \mathrm{~cm}^{-2}$, just above $n_{o}$, it is clear that a pronounced increase in gain occurs in the vicinity of the characteristic density.

The peak is less than an meV wide and very large in the absence of broadening effects. For $n>n_{o}$ and in the absence of broadening, the gain has a square-root singularity at the transparency edge. This divergence is associated with the flat-band regions on either side 
of the quasi-particle gaps shown in Fig. 1b. Because of the sensitivity of the peak height to broadening, the calculated threshold density is also sensitive to this effect. The threshold density corresponding to $100 \mathrm{~cm}^{-1}$ gain, the value cited in Refs. [1-3], is calculated to be $3.3 \times 10^{11} \mathrm{~cm}^{-2}$, and lies just $20 \%$ below $n_{o}$. In these experiments the typical excitation density by optical pumping was $5 . \times 10^{11} \mathrm{~cm}^{-2}$. The experimental threshold densities were estimated to be less than $7.3 \times 10^{11} \mathrm{~cm}^{-2}$, in agreement with the present estimates [1] 3].

The coherent exciton state may therefore provide an alternative explanation to the proposed inhomogeneous-linewidth theory of lasing in ZnSe/ZnCdSe quantum wells. Since this system is the first lasing semiconductor having excitons that are sufficiently robust at ordinary temperatures, these alternative explanations are both important. Both viewpoints lead to lasing below the exciton line. The coherent exciton state becomes important at the characteristic density $n_{o}$. Numerical calculations of the gap parameter characterizing this state yield results that bear qualitative similarity to experiment. An experimental attempt to measure the quasi-particle gap with IR radiation, akin to that first used to measure the superconducting gap [9], may be used to decide the issue. The experiment in this case, however, is far simpler since the magnitude of the gap is about $40 \mathrm{meV}$, which should be observable even in the presence of broadening effects using differential optical spectroscopy.

We are grateful to J. Ding, B.I. Halperin, P.C. Martin and A. Nurmikko for helpful discussions. This work was supported by an ARPA/URI subcontract through Brown University No. 283-250040 and by the U.S. Advanced Research Projects Agency (ARPA) through U.S. Office of Naval Research (ONR) Contract No. N00014-93-1-0549. 


\section{REFERENCES}

* Permanent address: Max-Planck-AG "Halbleitertheorie", Hausvogteiplatz 5-7, D-10117 Berlin, Germany.

[1] J. Ding, H. Jeon, T. Ishihara, A.V. Nurmikko, H. Luo, N. Samarth, J. Furdyna, Surf. Sci. 267, 616 (1992).

[2] J. Ding, H. Jeon, T. Ishihara, M. Hagerott, A.V. Nurmikko, H. Luo, N. Samarth, J. Furdyna, Phys. Rev. Lett. 69, 1707 (1992).

[3] J. Ding, M. Hagerott, T. Ishihara, H. Jeon, A.V. Nurmikko, Phys. Rev. B 47, 10528 (1993).

[4] See R. Zimmermann, Phys Stat Sol. B 76, 191 (1976).

[5] This ambiguity results from the representation of a two-particle excitation on a oneparticle energy diagram. Note that in the dilute limit the exciton binding energy is similarly divided equally between the constituent electron and hole. We also observe that this problem is theoretically related to that of a semiconductor driven by a coherent monochromatic light field, which has been treated by several authors (See Ref. [6] and V.M. Galitskiï, S.P. Goreslavskii, and V.F. Elesin, Zh. Eksp. Teor. Fiz. 57, 207 (1969) [Sov. Phys. - JETP 30, 117 (1970)]; T. Iida, Y. Hasegawa, H. Higashimura, M. Aihara, Phys. Rev. B 47, 9328 (1993).) and also to the excitonic insulator state (See, for example, B.I. Halperin and T.M. Rice, Solid State Physics vol. 21, p. 115 (F. Seitz, D. Turnbull and H. Ehrenreich, Eds.), (Academic Press, New York, 1968).). This relationship will be explored in a future publication.

[6] C. Comte, G. Mahler, Phys. Rev. B 34, 7164 (1986).

[7] P.M. Young, E. Runge, M. Ziegler, H. Ehrenreich, Phys. Rev. B 49, 7424 (1994).

[8] P.M. Young, P.M. Hui, H. Ehrenreich, Phys. Rev. B 44, 12969 (1991); P.M. Young, H. Ehrenreich, P.M. Hui, N.F. Johnson, J. Appl. Phys. 74, 7369 (1993). 
[9] M. Tinkham, Introduction to Superconductivity, (McGraw-Hill, New York, 1975). 


\section{FIGURES}

Noninteracting system (a) and interacting system (b) for the characteristic carrier density $n_{o}$. In the noninteracting case the quasi-Fermi levels of the conduction electrons and valence holes are $\mu_{e}$ and $\mu_{h}$ respectively. In the interacting case a quasi-particle gap forms at the conduction-electron and valence-hole Fermi surfaces. The gaps in the valence and conduction bands labelled $E_{e h} / 2$ each correspond to half the energy required to break up the exciton, one half being assigned to the electron and one half to the hole. The gain and absorption regions in the interacting system are indicated by the arrows marked "g" and "a" respectively. The gaps $E_{\text {eh }}$ produce a transparency regime, indicated by the arrow "tr". The characteristic density is defined as the density where $\mu_{e}+\mu_{h}=E_{G}$, and therefore the effective binding energy vanishes.

$E_{e h}$ and $2 \Delta$ as functions of density for $90 \AA, 30 \AA$, and strictly $2 \mathrm{D}$ quantum wells. $2 \Delta$ vanishes as the density approaches zero. At the characteristic density, when the effective binding energy of the exciton vanishes (at $4 \times 10^{11} \mathrm{~cm}^{-2}$ for the $90 \AA$ quantum well), $2 \Delta$ has become comparable to the isolated exciton binding energy.

Gain (positive) and absorption (negative) measured relative to $\mu_{e h}$ for three densities near the transition to the coherent state: $2.9 \times 10^{11} \mathrm{~cm}^{-2}, 3.7 \times 10^{11} \mathrm{~cm}^{-2}$ and $4.1 \times 10^{11}$ $\mathrm{cm}^{-2}$. A linewidth of $10 \mathrm{meV}$, as seen in experiments, has been introduced. At the lowest density the gain region is barely detectable. At the middle density the maximum gain is 200 $\mathrm{cm}^{-1}$. At the highest density, which is greater than the characteristic density, the gain has increased even more dramatically. In each case, there is a transparency region between the gain region and the absorption region. 
Characteristic Carrier Density $\mathrm{n}_{\mathrm{o}}$

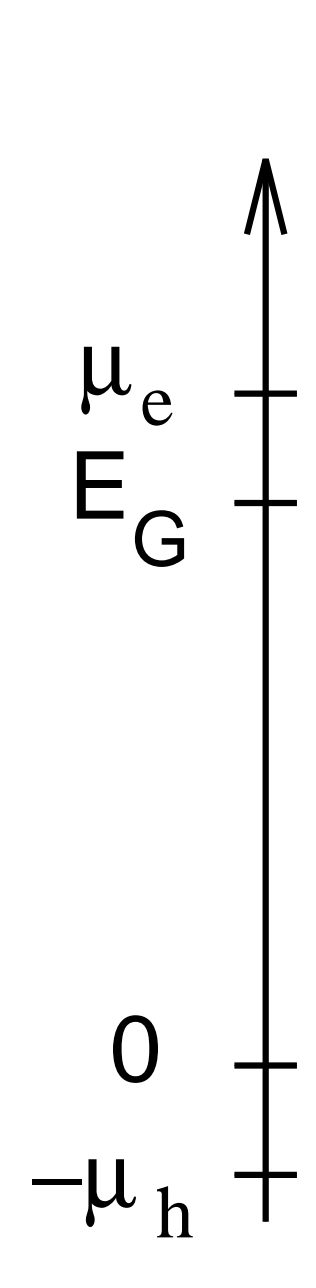

$$
V(q)=0
$$$$
V(q) \neq 0
$$

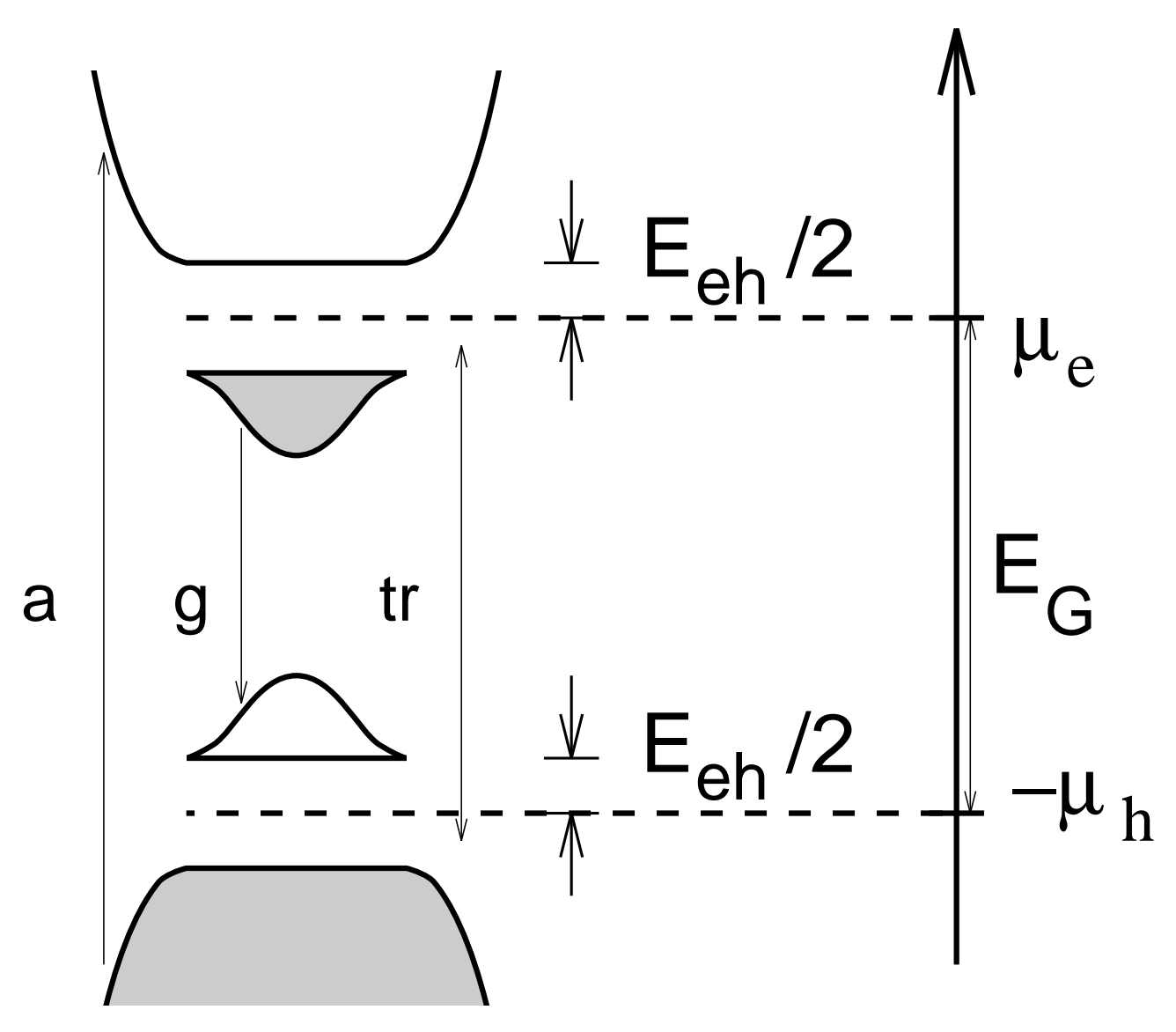

(a)

(b)

Figure 1 




Figure 2 


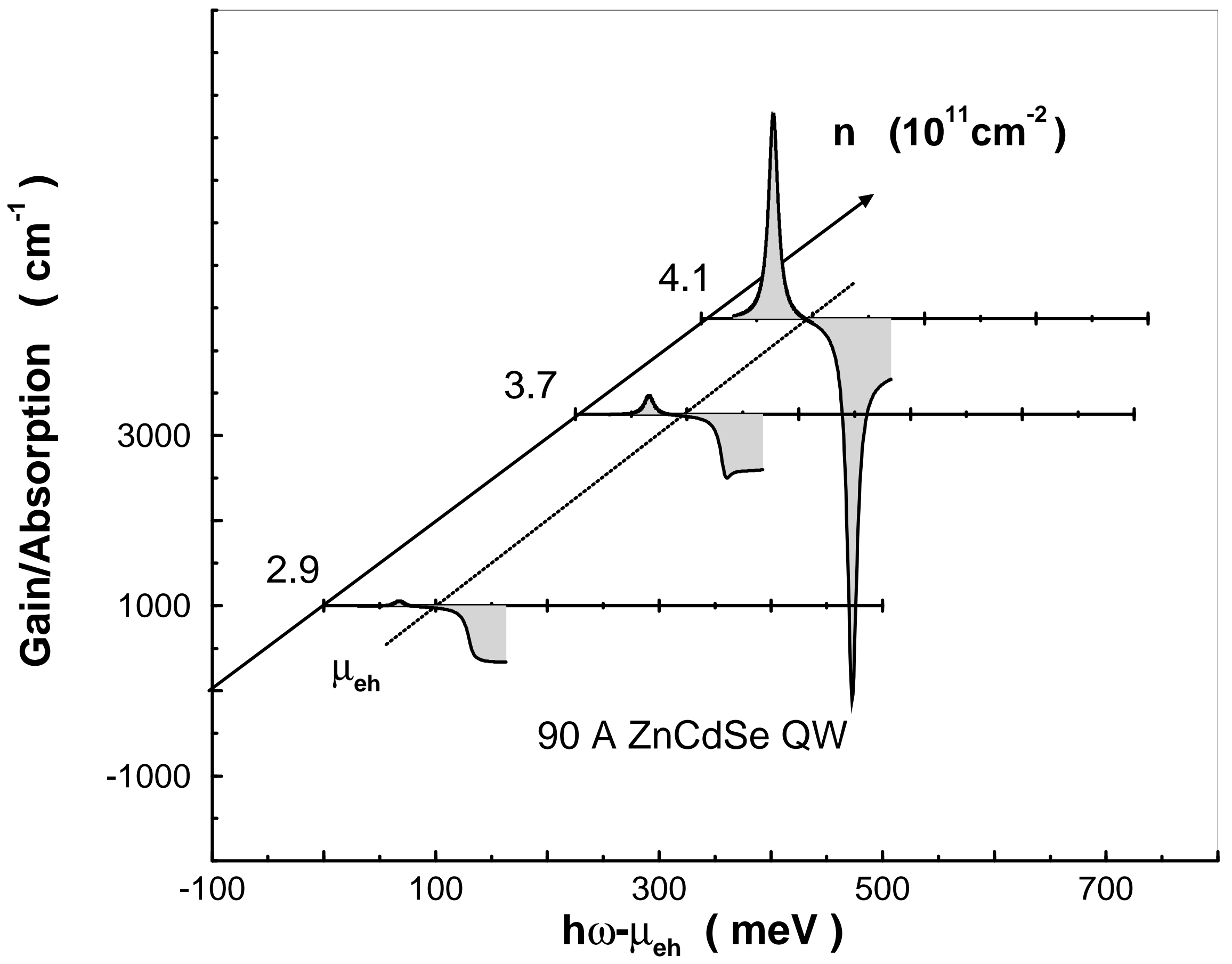

Figure 3 